\title{
Review Article \\ Metabolic and Physiological Roles of Branched-Chain Amino Acids
}

\author{
Md. Monirujjaman ${ }^{1}$ and Afroza Ferdouse ${ }^{2}$ \\ ${ }^{1}$ Human Nutritional Sciences, University of Manitoba, Winnipeg, MB, Canada R3E 0M2 \\ ${ }^{2}$ Biochemistry and Molecular Biology, University of Dhaka, Dhaka 1000, Bangladesh \\ Correspondence should be addressed to Md. Monirujjaman; monirujjaman108@gmail.com
}

Received 6 July 2014; Accepted 8 August 2014; Published 19 August 2014

Academic Editor: Haile Yancy

Copyright ( $2014 \mathrm{Md}$. Monirujjaman and A. Ferdouse. This is an open access article distributed under the Creative Commons Attribution License, which permits unrestricted use, distribution, and reproduction in any medium, provided the original work is properly cited.

\begin{abstract}
Branch chain amino acids (BCAAs) have unique properties with diverse physiological and metabolic roles. They have functions other than simple nutrition. Different diseases including metabolic disease lead to protein loss, especially muscle protein. Supplementation of BCAAs promotes protein synthesis and reduces break down, as well as improving disease conditions. They are important regulators of mTOR signaling pathway and regulate protein synthesis as well as protein turnover. BCAAs facilitate glucose uptake by liver and SK muscle and also enhance glycogen synthesis. Oxidation of BCAAs seems to be beneficial for metabolic health as their catabolism increases fatty acid oxidation and reduces risk of obesity. BCAAs are also important in immunity, brain function, and other physiological aspects of well-being. All three BCAAs are absolutely required for lymphocyte growth and proliferation. They are also important for proper immune cell function. BCAAs may influence brain protein synthesis, and production of energy and may influence synthesis of different neurotransmitters. BCAAs can be used therapeutically and future studies may be directed to investigating the diverse effects of BCAAs in different tissues and their signaling pathways.
\end{abstract}

\section{Introduction}

Amino acids (AAs) are biologically important compounds containing carboxylic and amine moiety as functional groups and are the building blocks of protein. They play important metabolic and physiological roles in all living organisms. Although more than 300 AAs have been found, only 20 AAs are known as standard AA and contain a specific sidechain specific to each AA [1]. Three branch chain amino acids (BCAAs), including isoleucine, leucine, and valine, have unique properties with diverse physiological and metabolic roles. BCAAs are primarily oxidized in the peripheral tissue, in particular in skeletal (SK) muscle, whereas the other AA catabolizes in the liver. BCAAs may regulate rate of protein synthesis and degradation in SK muscle and other organs. BCAAs and aromatic AA bind to the same carrier proteins to be transported to brain. They compete with each other and their ratio in brain may influence the synthesis of specific neurotransmitters, and that may influence the behavior of an organism $[2,3]$. BCAAs have glycogen sparing action and they have an opposite relation with tryptophan levels of brain, which is the precursor of serotonin. Serotonin is a mediator of central fatigue. Thus supplementation of BCAAs may prevent fatigue during extensive exercise, and diet enriched with BCAAs may improve muscle protein metabolism, body maintenance, and also aerobic exercise [3, 4]. BCAAs have functions other than simple nutrition. AAs supplementation enriched with BCAAs clinically is used for patients with liver diseases, renal failure, sepsis, and surgical injury [2, 5-7]. It is also found that BCAAs may affect gene expression, hepatocyte apoptosis and regeneration, and insulin resistance. They are necessary for lymphocyte proliferation and dendritic cell maturation, and they also inhibit cancer cell proliferation [8-13]. In this review we will provide some insights into metabolic and physiological roles of BCAAs.

\section{Metabolism and Metabolic Roles of BCAAs}

For metabolism, AAs are needed to be transported into the cell. Like glucose, AAs are also transported to the cell via special carrier mediated pathway. Inside the cell, 
transamination reaction of BCAAs gives rise to keto acids, specific to each AA. The keto acids further undergo oxidative decarboxylation to produce acyl-CoA derivatives and enter TCA cycle. The enzyme (activity) responsible for BCAA metabolism is found mostly in SK muscle, heart, and kidney but to lesser extent in liver [2].

In muscle, BCAAs not only provide nonspecific carbon source of oxidation for production of energy but also act as a precursor for muscle protein synthesis. BCAAs are more energy efficient than glucose. For example, complete oxidation of leucine in muscle produces more energy than complete oxidation of glucose in the form of ATP. Oxidation of BCAAs increases under various physiological conditions to meet the energy demand including the demand at starvation. It is also observed that BCAAs regulate the turnover of protein in muscles by inhibiting protein degradation and enhancing protein synthesis [14-16]. Several metabolic and clinical conditions, such as starvation, surgery, trauma, sepsis, liver disease, and cancer, lead to protein loss especially muscle protein and supplementation of BCAAs promotes protein synthesis and reduces break down as well as improving those conditions $[2,17-22]$. There is an increasing demand for AAs, alanine, and glutamine in those clinical conditions and they can be synthesized in SK muscle from BCAAs oxidation. So, BCAAs can cover the elevated demands for alanine and glutamine by the body during those clinical conditions [23].

2.1. Protein Synthesis and Turnover. Initiation of mRNA translation is one of the key steps in protein synthesis. Amino acids may stimulate protein synthesis by enhancing this important step of protein synthesis [24]. Deacylated tRNA reduces or inhibits protein synthesis by affecting the initiation step. BCAAs may lead to formation of aminoacyltRNA derivatives that enhance initiation of protein synthesis by minimizing that inhibitory effect. Another possible way that BCAAs may influence protein synthesis is the formation of active metabolic intermediate by the oxidation of BCAAs [25]. BCAAs have unique role in protein synthesis as well as in turnover. Buse et al. [26] studied in vitro effect of BCAAs on protein synthesis by analyzing polysome structures. Healthy rats were injected with BCAAs intravenously after 48-96 hours of fasting. And they observed that the number of polysomes (units) decreases during fasting state and supplementation of BCAAs or leucine alone significantly increases the density of polysomes, which was not observed when rat was fed normal food, which suggests that BCAAs, in particular leucine, enhances protein synthesis. In another study, Li and Jefferson [25] examined influence of BCAAs supplementation on protein turnover in rat SK muscle. Fasting young rats were supplemented with different combinations of BCAAs by perfusion. A significant increase (25-50\%) of muscle protein synthesis and significant decrease (30\%) in protein degradation were observed in perfused supplemented group compared to control. It was also observed that when the BCAAs were removed from complete mixture of amino acids, the rest of the amino acids showed no effects on protein turnover [25]. So, BCAAs not only enhance protein synthesis, but also influence protein turnover.
2.2. BCAAs and Signaling. Hypoalbuminemia and muscle wasting symptoms are quite common in liver cirrhotic patient. Oral administration of BCAA is found beneficial by increasing plasma albumin and reducing muscle wasting in both animals and humans. It is proposed that BCAAs accelerate protein synthesis in liver and other tissues via mammalian target of rapamycin (mTOR) signaling pathways. mTOR is a serine/threonine protein kinase and its signaling pathways may regulate protein synthesis and transcription as well as other cellular functions $[27,28]$. BCAA, particularly leucine, is the potent activator of mTOR signaling and activates protein synthesis by translational initiation [26]. In a study [29], rat hepatocytes in culture were incubated with different combinations of BCAAs and secretion of albumin was observed. It was found that mixture of BCAAs promotes albumin production in dose dependent manner and they were the key effectors. Rapamycin, which is the potent inhibitor of mTOR, reduces more than half of this production of albumin promoted by leucine. It was also observed that activation of downstream translational effector proteins of mTOR, elF4E-BP1, and p70 S6 kinase is induced by leucine only. And rapamycin completely inhibits those proteins. These observations suggest that synthesis of albumin in rat hepatocytes via mTOR signaling is promoted by BCAAs, particularly by leucine. In another study [30] starving male rats were orally administered either saline or each BCAA separately. Also, starved rats were injected intravenously with rapamycin before leucine administration. It was found that only leucine stimulated significant protein synthesis in SK muscle compared to controls. Moreover, leucine effectively enhanced phosphorylation and activation of elF4E-BP1 and p70 S6 kinase. Both in leucine-treated and starved rats, rapamycin inhibited protein synthesis.

All of these studies suggest that BCAAs, particularly, leucine, promote protein synthesis in SK muscle as well as in the liver by the same mechanism via mTOR.

2.3. Glucose Metabolism. Cirrhotic patients also suffer from impaired glucose metabolism and many of them develop diabetes mellitus. Synthesis of glycogen takes place in liver and SK muscles; they are the main reservoir of glycogen too. This glycogen store is very important in controlling blood glucose level. Cirrhotic patients have low level of glycogen store in their liver and SK muscle compared to healthy individuals [31]. BCAAs facilitate glucose uptake by liver and SK muscle as well as enhancing glycogen synthesis. Nishitani et al. [32] collected soleus muscles from healthy rats and incubated them with leucine in insulin-free conditions to test the effect of leucine on glucose uptake. They found that leucine promotes glucose uptake; moreover, $\alpha$-ketoisocaproic acid, a metabolic product of leucine, showed similar stimulatory effect. They observed that inhibition of phosphatidylinositol 3-kinase (PI3-kinase) or protein kinase C (PKC) by selective inhibitors leads to complete loss of stimulatory effect of leucine. However, rapamycin treatment showed no effect. These observations indicate that leucine stimulates glucose transport in SK muscle by insulin-independent manner through PKC and PI3-kinase pathways rather than mTOR pathway. In another study Peyrollier et al. [33] deprived 
myoblast L6 cells $\sim 1 \mathrm{~h}$ for AAs and after that incubated them with leucine. They observed a significant increase of PI3kinase and p70 S6 kinase activity. They also found that glycogen synthase kinase-3 (GSK-3) is inactivated by leucine. GSK3 is an enzyme that inactivates glycogen synthase (GS) activity and stops glycogen synthesis. These results suggest that BCAA supplementation may enhance glycogen synthesis by activating GS.

2.4. Energy Metabolism. There is an inverse relationship between physical activity and obesity. Obesity on the other hand is associated with different types of complications, including metabolic diseases. Regular physical activities keep a person fit with reducing risks of those diseases. It is beneficial and important for us to know how the energy metabolism is regulated and coordinated. BCAAs oxidation seems to be beneficial for our metabolic health as their catabolism increases fatty acid oxidation as well as reducing risk of obesity. There are higher levels of plasma BCAAs found in obese animals and humans; on the other hand, lower levels of plasma BCAAs are found in lean and exercised animals and humans. It is also found that supplementation of BCAAs enriched protein is beneficial in animals and humans by increasing exercise performance, composition of body protein and properties, and better glucose tolerance and control; all of these are related to better health and fitness [4]. Nishimura et al. [34] fed mice with high fat diet and supplemented isoleucine to test the effect of isoleucine supplementation on obesity condition, glucose, and fat homeostasis. They observed that isoleucine supplementation leads to a decrease in weight gain and reduced lipid mass in isoleucine supplemental group compared to control. Triglyceride concentrations and degree of hyperinsulinemia were also lower in hepatic and SK muscle of isoleucine supplemented group. In a double-blind, placebo-control, cross-over study [35], human volunteers were supplemented with either the BCAA or the placebo for 3 days. Their exercise capacity and lipid oxidation were measured. It was found that BCAA supplementation increases lipid oxidation during exercise and helps to overcome fatigue condition. The glucose metabolism of the volunteer also improved. Qin et al. [36] showed that there is an inverse relation between BCAA intake and obesity. Apparent middle aged healthy adults from China, Japan, UK, and USA, who consumed higher amount of BCAAs, had low incidence of obesity and overweight status. All of these evidences suggest that BCAAs have large influence on energy metabolism as well as reducing risks of obesity.

\section{Physiological Roles of BCAAs}

3.1. BCAAs in Immunity. The immune system is important in protecting host from pathogens (including bacteria, viruses, fungi, parasites, etc.), existing in surrounding environments, and also protects from other notorious threats. Immune cells (i.e., lymphocytes, neutrophils, etc.) express dehydrogenase and decarboxylase enzyme and can effectively oxidize BCAAs [37-39]. All three BCAAs are absolutely required for lymphocytes growth and proliferation. BCAAs are also important for cytotoxic T lymphocyte and natural killer cell activity [40].
In a study [41] mice were supplemented with diet containing optimal level of protein or diet containing individual AA, except for limited amount of BCAAs. The susceptibility to Salmonella typhimurium infection and immune activity were measured. They found a higher mortality rate to $S$. typhimurium when mice were fed diet containing restricted amount of BCAAs (any). The liver and spleen were also colonized with higher number of S. typhimurium in that group of mice. BCAA restriction also leads to lower number of spleen cells and less antibody titer against $S$. typhimurium in the serum. These results suggest that restriction of any BCAA may impair host defense system. Human study [42] with surgery patients shows that higher BCAA supplementation leads to higher postsurgery blood lymphocytes, higher immune parameters, and better recovery. Bassit et al. [43] supplemented BCAAs to male elite triathletes to measure the effects of that supplementation on immunity parameters. They observed that BCAAs supplementation increased lymphocyte proliferation and modified the pattern of cytokine production and shift of the immune response from Th2 to Th1, which is beneficial for the athletes.

3.2. BCAAs in Sepsis. Sepsis is a physiological condition in which severe inflammation occurs in whole body due to infection and it may be life threatening [44]. Potential beneficial roles of BCAAs in sepsis have been studied. Mori et al. [5] supplemented total parenteral nutrition (TPN) enriched with branched-chain amino acids (BCAAs) to septic rats. They found that BCAA-enriched TPN leads to significant improvement of nitrogen balance and decreases mortality compared to conventional TPN in septic rats. In a randomized, multicenter study [45] sepsis patients were supplemented TPN, enriched with BCAAs or conventional TPN only. There was significant decrease in mortality and improvements of visceral proteins half-life were observed in the BCAA-supplemental group. These studies suggest that BCAAs have a beneficial effect in septic patients.

3.3. BCAAs and Diabetes. Diabetes is one of the most prevalent diseases worldwide and is associated with several factors such as age, race, body weight, and food habit. There is a strong correlation between circulating BCAAs and diabetes prediction [46]. In a recent study it was found that patients with type 2 diabetes, who had impaired fasting glucose, also had elevated levels of BCAAs compared to healthy individuals [47]. Mammalian target of rapamycin complex 1 (mTORC1) is a nutrient-sensitive kinase, important for growth and proliferation of beta cell as well as insulin secretion. BCAAs are important regulators of mTORC1 signaling and elevated levels of plasma BCAAs for a longer period can cause hyperactivation of mTOR signaling. And that may lead to early beta cell dysfunction and destruction [48]. So, it is important to maintain normal plasma levels of BCAA.

3.4. BCAAs in Brain Functions. BCAAs may also play important roles in brain function. BCAAs may influence brain protein synthesis and production of energy and may influence synthesis of different neurotransmitters, that is, serotonin, 
dopamine, norepinephrine, and so forth, directly or indirectly. Major portion of dietary BCAAs is not metabolized by liver and comes into systemic circulation after a meal. BCAAs and aromatic AA, such as tryptophan (Trp), tyrosine (Tyr), and phenylalanine (Phe), share the same transporter protein to transport into brain. Trp is the precursor of neurotransmitter serotonin; Tyr and Phe are precursors of catecholamines (dopamine, norepinephrine, and epinephrine). When plasma concentration of BCAAs increases, the brain absorption of BCAAs also increases with subsequent reduction of aromatic AA absorption. That may lead to decrease in synthesis of these related neurotransmitters [3]. Catecholamines are important in lowering blood pressure. When hypertensive rats were injected with Tyr, their blood pressure dropped markedly and injection with equimolar amount of valine blocks that action [49]. In vigorous working persons, such as in athletes, depletion of muscle and plasma BCAAs is normal. And that depletion of muscle and plasma BCAAs may lead to increase in Trp uptake by brain and release of serotonin. Serotonin on the other hand leads to central fatigue. So, supplementation of BCAAs to vigorously working person may be beneficial for their performance and body maintenance [3].

\section{Conclusions}

BCAAs have unique characteristics as they are not primarily oxidized in liver and they regulate protein synthesis and degradation in muscle as well as other tissues. In addition, BCAAs compete with aromatic AA to enter into brain. They have diverse metabolic and physiological roles. BCAAs are also important regulators of mTOR signaling that regulates protein and glycogen metabolism in liver and SK muscles. These effects in liver and SK muscles are important in maintaining body composition and glucose balance. They are also important regulators of neurotransmitters in brain. So, BCAAs may be therapeutically useable in various neurological disorders. However, more research is needed in this field. In vitro and in vivo studies of BCAAs suggest that they might have beneficial effects in various disorders. It is important and necessary to explore whether BCAAs have other therapeutic effects on other tissues. Future investigations may be directed to fully understand the diverse effects of BCAA in different tissues and associated signaling pathways.

\section{Conflict of Interests}

The authors declare that there is no conflict of interests regarding the publication of this paper.

\section{References}

[1] G. Wu, "Amino acids: metabolism, functions, and nutrition," Amino Acids, vol. 37, no. 1, pp. 1-17, 2009.

[2] B. Skeie, V. Kvetan, K. M. Gil, M. M. Rothkopf, E. A. Newsholme, and J. Askanazi, "Branch-chain amino acids: their metabolism and clinical utility," Critical Care Medicine, vol. 18, no. 5, pp. 549-571, 1990.
[3] J. D. Fernstrom, "Branched-chain amino acids and brain function," Journal of Nutrition, vol. 135, supplement 6, pp. 1539S1546S, 2005.

[4] H. Kainulainen, J. J. Hulmi, and U. M. Kujala, "Potential role of branched-chain amino acid catabolism in regulating fat oxidation," Exercise and Sport Sciences Reviews, vol. 41, no. 48, pp. 194-200, 2013.

[5] E. Mori, M. Hasebe, and K. Kobayashi, "Effect of total parenteral nutrition enriched in branched-chain amino acids on metabolite levels in septic rats," Metabolism, vol. 37, no. 9, pp. 824-830, 1988.

[6] G. L. Blackburn, L. L. Moldawer, S. Usui, A. Bothe Jr., S. J. D. O'Keefe, and B. R. Bistrian, "Branched chain amino acid administration and metabolism during starvation, injury, and infection," Surgery, vol. 86, no. 2, pp. 307-315, 1979.

[7] H. Mochizuki, O. Trocki, L. Dominioni, and J. W. Alexander, "Effect of a diet rich in branched chain amino acids on severely burned guinea pigs," Journal of Trauma, vol. 26, no. 12, pp. 1077$1085,1986$.

[8] K. Tajiri and Y. Shimizu, "Branched-chain amino acids in liver diseases," World Journal of Gastroenterology, vol. 19, no. 43, pp. 7620-7629, 2013.

[9] K. Sugiyama, L. Yu, and N. Nagasue, "Direct effect of branchedchain amino acids on the growth and metabolism of cultured human hepatocellular carcinoma cells," Nutrition and Cancer, vol. 31, no. 1, pp. 62-68, 1998.

[10] T. Kawaguchi, Y. Nagao, H. Matsuoka, T. Ide, and M. Sata, "Branched-chain amino acid-enriched supplementation improves insulin resistance in patients with chronic liver disease," International Journal of Molecular Medicine, vol. 22, no. 1, pp. 105-112, 2008.

[11] M. Arakawa, T. Masaki, J. Nishimura, M. Seike, and H. Yoshimatsu, "The effects of branched-chain amino acid granules on the accumulation of tissue triglycerides and uncoupling proteins in diet-induced obese mice," Endocrine Journal, vol. 58, no. 3, pp. 161-170, 2011.

[12] M. Kuwahata, H. Kubota, H. Kanouchi et al., "Supplementation with branched-chain amino acids attenuates hepatic apoptosis in rats with chronic liver disease," Nutrition Research, vol. 32, no. 7, pp. 522-529, 2012.

[13] K. Ichikawa, T. Okabayashi, Y. Shima et al., "Branched-chain amino acid-enriched nutrients stimulate antioxidant DNA repair in a rat model of liver injury induced by carbon tetrachloride," Molecular Biology Reports, vol. 39, no. 12, pp. 1080310810, 2012.

[14] H. R. Freund and M. Hanani, "The metabolic role of branchedchain amino acids," Nutrition, vol. 18, no. 3, pp. 287-288, 2002.

[15] A. L. Goldberg and R. Odessey, "Oxidation of amino acids by diaphragms from fed and fasted rats." The American Journal of Physiology, vol. 223, no. 6, pp. 1384-1391, 1972.

[16] M. G. Buse and S. S. Reid, "Leucine: a possible regulator of protein turnover in muscle," Journal of Clinical Investigation, vol. 56, no. 5, pp. $1250-1261,1975$.

[17] J. de Bandt and L. Cynober, "Therapeutic use of branched-chain amino acids in burn, trauma, and sepsis," Journal of Nutrition, vol. 136, supplement, no. 1, pp. 308S-313S, 2006.

[18] E. Blomstrand, "Potential role of branched-chain amino acid catabolism in regulating fat oxidation," Journal of Nutrition, vol. 41, no. 4, pp. 194-200, 2006. 
[19] S. Khanna and S. Gopalan, "Role of branched-chain amino acids in liver disease: the evidence for and against," Current Opinion in Clinical Nutrition and Metabolic Care, vol. 10, no. 3, pp. 297303, 2007.

[20] T. M. O'Connell, “The complex role of branched chain amino acids in diabetes and cancer," Metabolites, vol. 3, no. 4, pp. 931945, 2013.

[21] R. Odessey and B. Parr, "Effect of insulin and leucine on protein turnover in rat soleus muscle after burn injury," Metabolism, vol. 31, no. 1, pp. 82-87, 1982.

[22] J. C. Manelli, M. Garabedian, N. Ounis, M. Houvenaeghel, A. Ottomani, and J. Bimar, "Effects on muscular and general proteolysis in burn patients of a solution enriched with branched amino acids," Annales Françaises d'Anesthésie et de Réanimation, vol. 3, no. 4, pp. 256-260, 1984.

[23] M. Holeček, "Glutamine and branched-chain amino acidspractical importance of their metabolic relations," Casopis Lekaru Ceskych, vol. 144, no. 3, pp. S9-S12, 2005.

[24] S. R. Kimball and L. S. Jefferson, "Regulation of protein synthesis by branched-chain amino acids," Current Opinion in Clinical Nutrition and Metabolic Care, vol. 4, no. 1, pp. 39-43, 2001.

[25] J. B. Li and L. S. Jefferson, "Influence of amino acid availability on protein turnover in perfused skeletal muscle," Biochimica et Biophysica Acta, vol. 544, no. 2, pp. 351-359, 1978.

[26] M. G. Buse, R. Atwell, and V. Mancusi, "In vitro effect of branched chain amino acids on the ribosomal cycle in muscles of fasted rats," Hormone and Metabolic Research, vol. 11, no. 4, pp. 289-292, 1979.

[27] E. J. Brown, M. W. Albers, K. Ichikawa, C. T. Keith, W. S. Lane, and S. L. Schreiber, "A mammalian protein targeted by G1-arresting rapamycin-receptor complex," Nature, vol. 369, no. 6483, pp. 756-758, 1994.

[28] N. Hay and N. Sonenberg, "Upstream and downstream of mTOR," Genes and Development, vol. 18, no. 16, pp. 1926-1945, 2004.

[29] C. Ijichi, T. Matsumura, T. Tsuji, and Y. Eto, "Branched-chain amino acids promote albumin synthesis in rat primary hepatocytes through the mTOR signal transduction system," Biochemical and Biophysical Research Communications, vol. 303, no. 1, pp. 59-64, 2003.

[30] J. C. Anthony, F. Yoshizawa, T. G. Anthony, T. C. Vary, L. S. Jefferson, and S. R. Kimball, "Leucine stimulates translation initiation skeletal muscle of postabsorptive rats via a rapamycinsensitive pathway," Journal of Nutrition, vol. 130, no. 10, pp. 24132419, 2000.

[31] S. Nishitani, C. Ijichi, K. Takehana, S. Fujitani, and I. Sonaka, "Pharmacological activities of branched-chain amino acids: specificity of tissue and signal transduction," Biochemical and Biophysical Research Communications, vol. 313, no. 2, pp. 387389, 2004.

[32] S. Nishitani, T. Matsumura, S. Fujitani, I. Sonaka, Y. Miura, and K. Yagasaki, "Leucine promotes glucose uptake in skeletal muscles of rats," Biochemical and Biophysical Research Communications, vol. 299, no. 5, pp. 693-696, 2002.

[33] K. Peyrollier, E. Hajduch, A. S. Blair, R. Hyde, and H. S. Hundal, "L- leucine availability regulates phosphatidylinositol 3-kinase, p70 S6 kinase and glycogen synthase kinase-3 activity in L6 muscle cells: evidence for the involvement of the mammalian target of rapamycin ( mTOR ) pathway in the L- leucine induced up-regulation of system A amino acid transport," The Biochemical Journal, vol. 350, part 2, pp. 361-368, 2000.

[34] J. Nishimura, T. Masaki, M. Arakawa, M. Seike, and H. Yoshimatsu, "Isoleucine prevents the accumulation of tissue triglycerides and upregulates the expression of PPAR $\gamma$ and uncoupling protein in diet-induced obese mice," Journal of Nutrition, vol. 140, no. 3, pp. 496-500, 2010.

[35] A. B. Gualano, T. Bozza, P. de Lopes Campos et al., "Branchedchain amino acids supplementation enhances exercise capacity and lipid oxidation during endurance exercise after muscle glycogen depletion," Journal of Sports Medicine and Physical Fitness, vol. 51, no. 1, pp. 82-88, 2011.

[36] L. Q. Qin, P. Xun, D. Bujnowski et al., "Higher branched-chain amino acid intake is associated with a lower prevalence of being overweight or obese in middle-aged East Asian and Western adults," Journal of Nutrition, vol. 141, no. 2, pp. 249-254, 2011.

[37] S. D. Skaper, D. P. Molden, and J. E. Seegmiller, "Maple syrup urine disease: branched-chain amino acid concentrations and metabolism in cultured human lymphoblasts," Biochemical Genetics, vol. 14, no. 7-8, pp. 527-539, 1976.

[38] G. Schafer and P. Schauder, "Assessment of effects of amino acids and branched chain keto acids on leucine oxidation in human lymphocytes," Scandinavian Journal of Clinical \& Laboratory Investigation, vol. 48, no. 6, pp. 531-536, 1988.

[39] P. Schauder and G. Schafer, "Oxidation of leucine in human lymphocytes," Scandinavian Journal of Clinical and Laboratory Investigation, vol. 47, no. 5, pp. 447-453, 1987.

[40] P. C. Calder, "Branched-chain amino acids and immunity", The Journal of Nutrition, vol. 136, supplement 1, no. 1, pp. 288S-293S, 2006.

[41] T. M. Petro and J. K. Bhattacharjee, "Effect of dietary essential amino acid limitations upon the susceptibility to Salmonella typhimurium and the effect upon humoral and cellular immune responses in mice," Infection and Immunity, vol. 32, no. 1, pp. 251-259, 1981.

[42] F. B. Cerra, J. E. Mazuski, E. Chute et al., "Branched chain metabolic support. A prospective randomized, double-blind trial in surgical stress," Annals of Surgery, vol. 199, no. 3, pp. 286291, 1984

[43] R. A. Bassit, L. A. Sawada, R. F. P. Bacurau et al., "Branchedchain amino acid supplementation and the immune response of long-distance athletes," Nutrition, vol. 18, no. 5, pp. 376-379, 2002.

[44] M. M. Levy, M. P. Fink, J. C. Marshall et al., "2001 SCCM/ ESICM/ACCP/ATS/SIS international sepsis definitions conference," Critical Care Medicine, vol. 31, no. 4, pp. 1250-1256, 2003.

[45] A. García-de-Lorenzo, C. Ortiz-Leyba, M. Planas et al., "Parenteral administration of different amounts of branch-chain amino acids in septic patients: clinical and metabolic aspects," Critical Care Medicine, vol. 25, no. 3, pp. 418-424, 1997.

[46] B. C. Batch, K. Hyland, and L. P. Svetkey, "Branch chain amino acids: biomarkers of health and disease," Current Opinion in Clinical Nutrition and Metabolic Care, vol. 17, no. 1, pp. 86-89, 2014.

[47] F. Xu, S. Tavintharan, C. F. Sum, K. Woon, S. C. Lim, and C. N. Ong, "Metabolic signature shift in type 2 diabetes mellitus revealed by mass spectrometry-based metabolomics," Journal 
of Clinical Endocrinology and Metabolism, vol. 98, no. 6, pp. E1060-E1065, 2013.

[48] B. C. Melnik, "Leucine signaling in the pathogenesis of type 2 diabetes and obesity," World Journal of Diabetes, vol. 3, no. 3, pp. 38-53, 2012.

[49] A. F. Sved, J. D. Fernstrom, and R. J. Wurtman, "Tyrosine administration reduces blood pressure and enhances brain norepinephrine release in spontaneously hypertensive rats," Proceedings of the National Academy of Sciences of the United States of America, vol. 76, no. 7, pp. 3511-3514, 1979. 

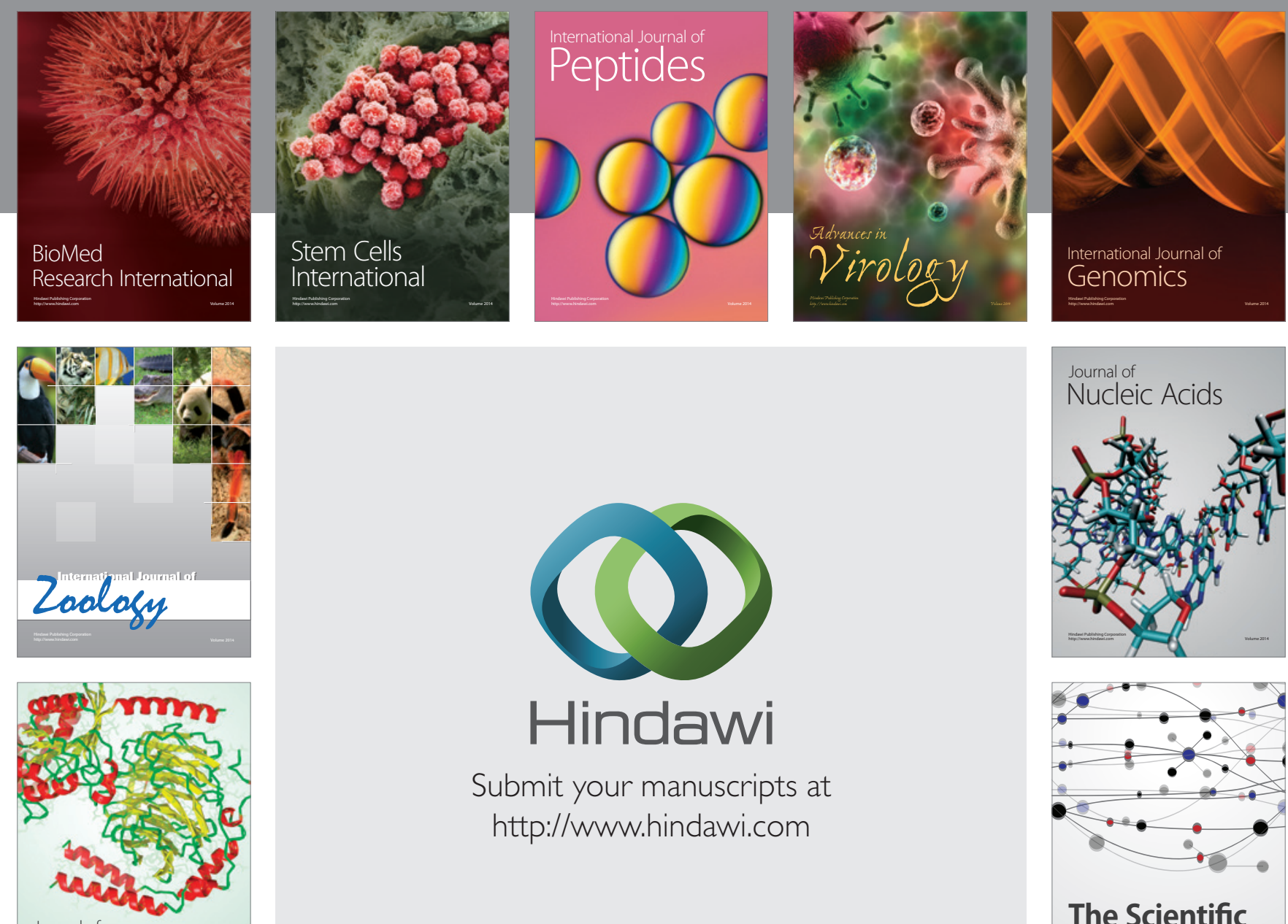

Submit your manuscripts at

http://www.hindawi.com

Journal of
Signal Transduction
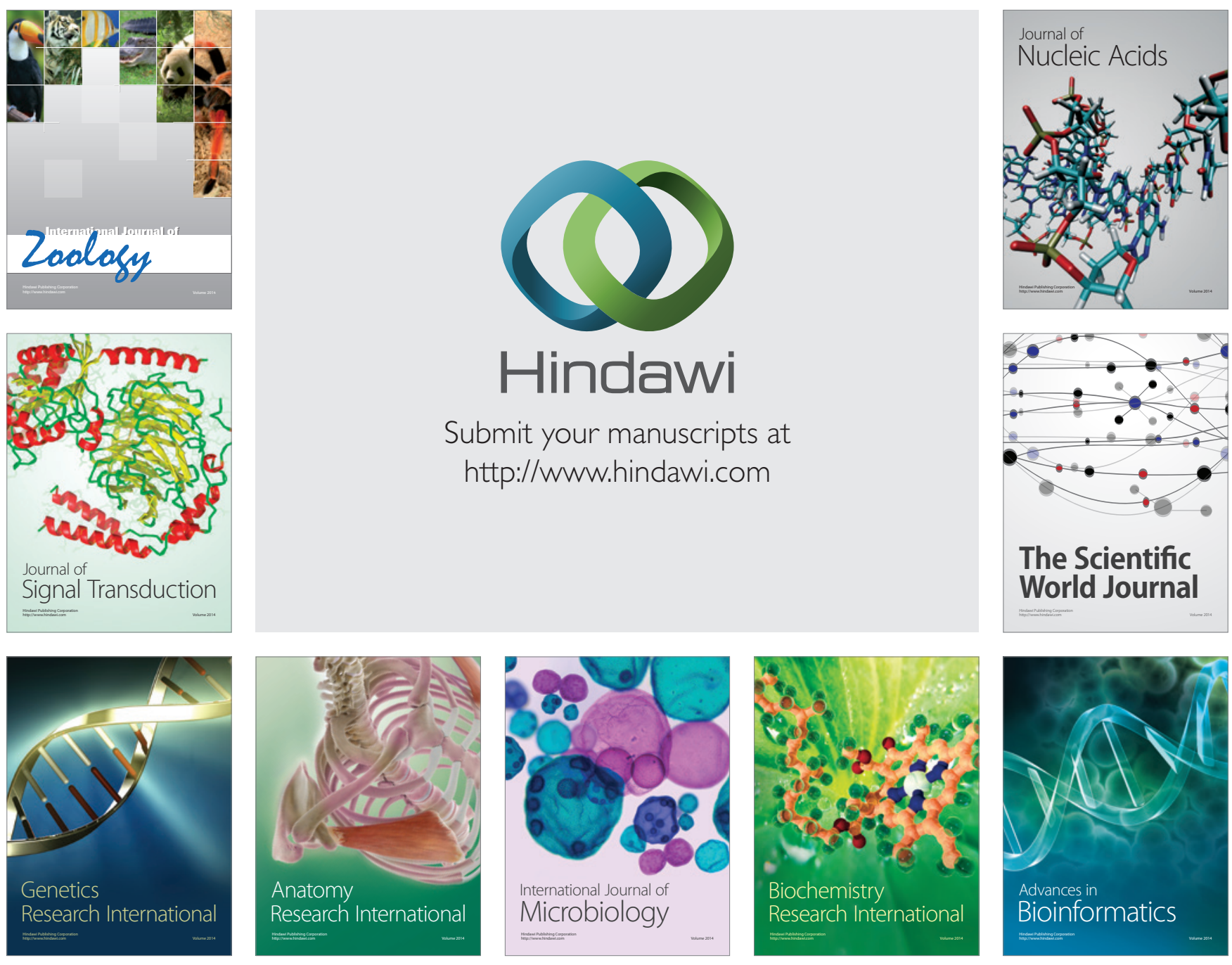

The Scientific World Journal
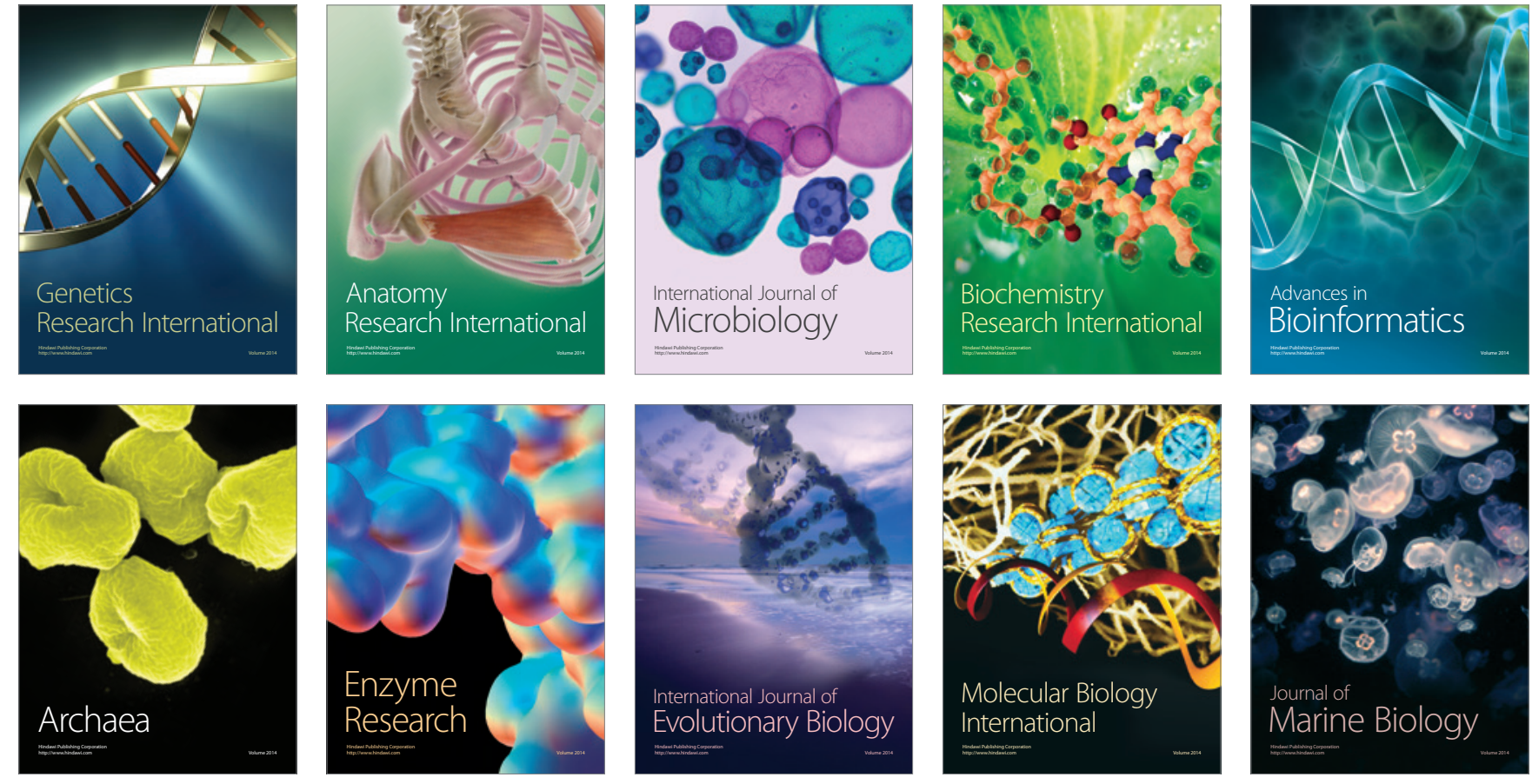